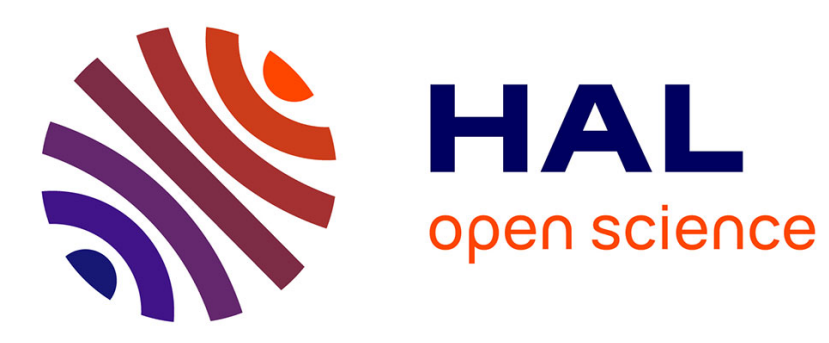

\title{
Bucarest, vivre et faire la ville entre héritages et mimétisme
}

Samuel Rufat, Bogdan Suditu

\section{To cite this version:}

Samuel Rufat, Bogdan Suditu. Bucarest, vivre et faire la ville entre héritages et mimétisme. Espace Géographique, 2008, 37 (4), pp.327-337. 10.3917/eg.374.0327 . hal-00596683

\section{HAL Id: hal-00596683 \\ https://hal.science/hal-00596683}

Submitted on 29 May 2011

HAL is a multi-disciplinary open access archive for the deposit and dissemination of scientific research documents, whether they are published or not. The documents may come from teaching and research institutions in France or abroad, or from public or private research centers.
L'archive ouverte pluridisciplinaire HAL, est destinée au dépôt et à la diffusion de documents scientifiques de niveau recherche, publiés ou non, émanant des établissements d'enseignement et de recherche français ou étrangers, des laboratoires publics ou privés. 


\section{Bucarest, vivre et faire la ville entre héritages et mimétisme}

Samuel RUFAT, Université de Lyon, UMR 5600

Bogdan SUDITU, Université de Bucarest

Bucarest, capitale macrocéphalique de près de 2 millions d'habitants, aspire à un rôle majeur dans l'Europe du Sud Est. Elle a dû surmonter ces dernières années les conséquences de l'urbanisme de Ceausescu. Une partie de ces chantiers sont restés suspendus : le centre ville est éventré, des structures en béton semblent encore aujourd'hui figées dans le temps, et les grues rouillent sur pied, puis tombent. Les destructions ont été concentrées le long des nouveaux axes; derrière les bâtiments socialistes les tissus anciens et les églises déplacées restent à l'abri des regards. En fait, deux villes se superposent à Bucarest depuis le milieu du XIX ${ }^{\text {ème }}$ siècle et l'avènement de son statut de capitale de la Roumanie. L'élite urbaine a cherché à éradiquer la ville "orientale " et à la remplacer par une ville dense, monumentale, moderne. Mais l'afflux de ruraux et l'autoconstruction traditionnelle ont perpétué ses charmes à la fois élégants et villageois, avec des maisons en bois et en torchis, aux marges de la légalité, privées de toute viabilité et parsemées de terrains vagues, d'animaux et de palissades. Bucarest apparaît tiraillée par ces héritages entre les différentes étapes d'une recherche de la modernité. La réutilisation de l'axe Est-Ouest percé par Ceausescu illustre bien ces ambiguïtés. D'un côté de cet axe, le Palais a été achevé pour concentrer le Parlement et les ministères, suivant le projet initial, de l'autre côté de l'axe, les façades des bâtiments destinés aux cadres du Parti ont été modifiées selon le style international pour accueillir banques et assurances. La capitale continue à faire peau neuve pour concurrencer les capitales européennes.

Après 1989, la période de "transition" devait se caractériser par la résorption des dysfonctionnements et l'articulation des différentes strates urbaines. L'entrée dans I'UE a été attendue comme leur aboutissement ; face à l'Université une horloge a décompté les jours jusqu'au $1^{\text {er }}$ janvier 2007. Mais la «transition » n'est pas achevée, les litiges issus des restitutions ont renforcé la prégnance des héritages tout en limitant les nouveaux projets. Bucarest a perdu un dixième de sa population de plus en plus vieillissante, son attractivité pourrait apparaître réduite alors que les enjeux du remodelage de l'espace urbain demandent des efforts considérables. Après la "Révolution ", Bucarest semblait à réinventer, aujourd'hui, les hésitations entre l'assimilation de ses héritages et un mimétisme ordinaire amplifient la lenteur de certaines mutations. Les principaux projets peinent à se matérialiser pendant que les tours de bureaux surgissent au hasard des opportunités. Comme la majorité de ses habitants, Bucarest est captive de la spéculation foncière, elle semble redevenue chaotique, ingouvernable.

\section{Loin de la table rase, le capharnaüm de l'habitat urbain}

Malgré les destructions récurrentes ${ }^{1}$, les tissus anciens n'ont pas disparu. Les destructions de la Seconde Guerre mondiale ont été moins importantes qu'à Varsovie ou à Sofia. Mais, après le séisme de 1977 celles qui ont fait place au Centre Civique ont entraîné la disparition d'un cinquième

\footnotetext{
${ }^{1}$ Dana Harhoiu fait de Bucarest l'archétype de la ville martyrisée, HARHOIU D., 1997, Bucarest, une ville entre Orient et Occident, Simetria-Arcub, Bucarest, p. 18
} 
du centre historique (environ 400 ha). Bucarest présente peu de monuments anciens, ses tissus témoignent en revanche de nombreuses opérations d'urbanisme qui se sont superposées du fait de changements de référents. Ce sont les Russes qui ont appliqué les principes de l'hygiénisme dans les années 1830, les architectes se sont tournés ensuite vers Haussmann, avant de se voir imposer le modèle soviétique. Dans les années 1980, la surimposition du Centre Civique et du nouvel axe EstOuest sur l'ancien quartier Uranus se fait sur le modèle de Pyongyang ${ }^{2}$. Pourtant, I'ancien " conglomérat de villages", est encore perceptible, comme le notait déjà Vintila Mihailescu en $1914^{3}$. La ville orientale reste présente au travers de la trame viaire, aux rues étroites, chaotiques et sinueuses, des constructions basses séparées par des friches, des petites églises orthodoxes et des caravansérails. De part et d'autre des percées du XIX ${ }^{\text {ème }}$ et du XXème siècle, les quartiers résidentiels sont restés en l'état. Quand aux percées les plus récentes, elles sont bordées de barres socialistes qui ceinturent les tissus anciens. Au cours des deux derniers siècles, le passage du modèle oriental à l'occidental produit d'un part une structure urbaine qui semble sans cesse inachevée, d'autre part des rues où les conceptions d'urbanisme et les styles différents sont juxtaposés. Le télescopage de ces cycles de destruction/reconstruction donne à la capitale un aspect chaotique.

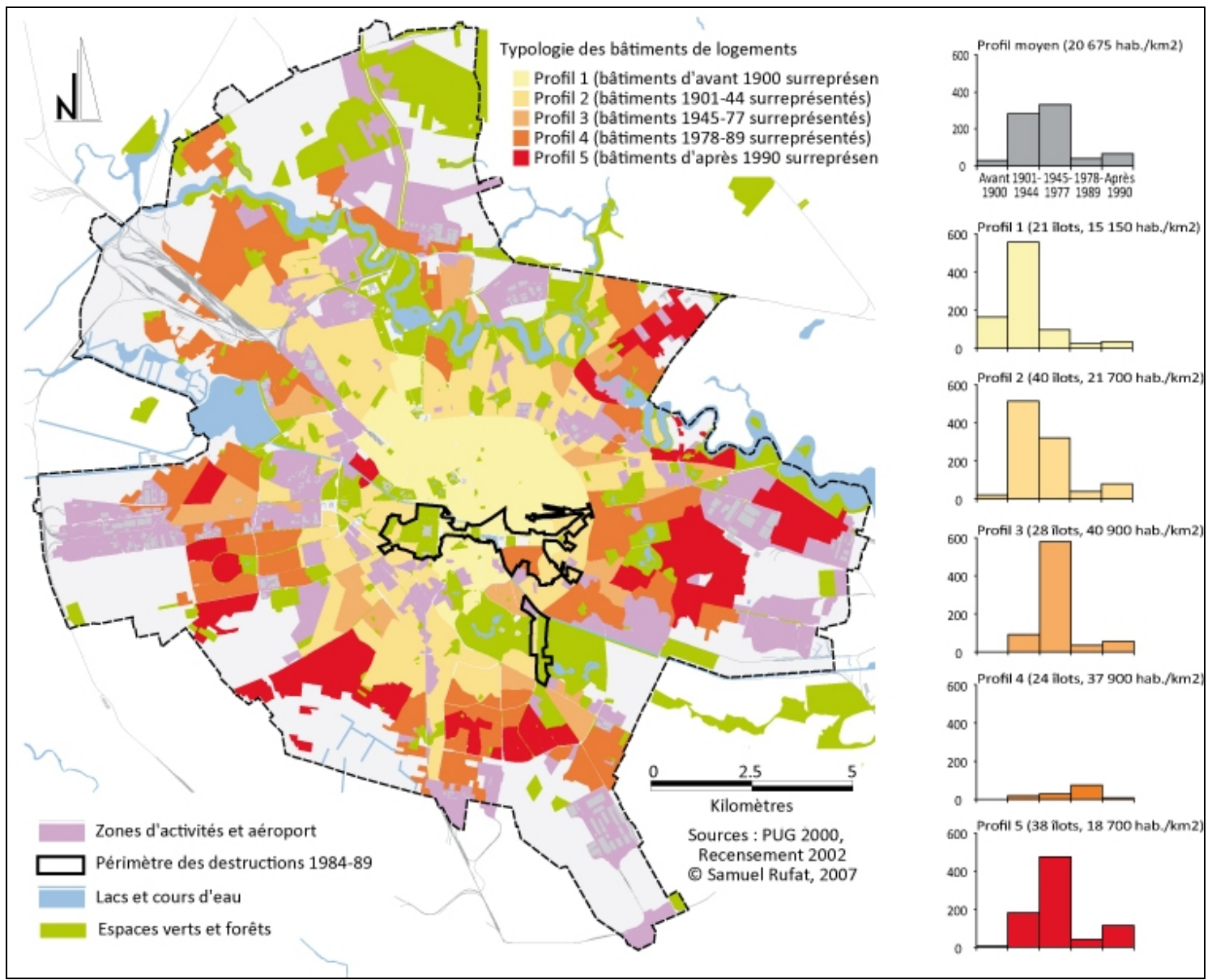

Carte 1 : Typologie des quartiers selon la date de construction des logements en 2002

Aujourd'hui, le parc immobilier reste dominé par les bâtiments anciens et l'habitat collectif. Plus de $40 \%$ des logements sont antérieurs à 1944 et près de $80 \%$ antérieurs aux importantes

\footnotetext{
2 Darrick Danta, 1993, «Ceaucescu's Bucharest », Geographiacl Review, vol. 83, n², pp. 170-182

${ }^{3}$ Minallescu V., 2003, Bucureşti. Evoluția geografică a unui oraş, édition de manuscrits de 1914 et 1977, Paideia, Bucarest, p. 
opérations menées après le séisme de 1977. Les bâtiments d'habitat collectif concentrent l'essentiel des logements (85\%). Ce parc immobilier traduit la différenciation de l'espace bucarestois et révèle les formes d'appropriation de la ville (carte 1).

La zone centrale est marquée par les logements individuels anciens, dont la majorité sont bâtis avant 1914, ponctués d'immeubles de l'Entre-deux-guerres. Le régime communiste a délaissé ces tissus qui se sont rapidement dégradés. Le centre et le Sud de cette zone ont été radicalement modifiés par la percée du nouvel axe Est-Ouest et la construction du Centre Civique, c'est le périmètre de logements collectifs les plus récent. Dans les années 1990, le centre retrouve son attractivité, accueille de nouveaux services et des tours de bureaux. Une série d'opérations ponctuelles et privées transforment le parc de logements. Au tournant du XXI ${ }^{\text {ème }}$ siècle, les réhabilitations marquent la valorisation du centre ville.

Entre les quartiers centraux et les grands ensembles, les zones intermédiaires sont caractérisées par les lotissements de l'Entre-deux-guerres : au Nord et à l'Ouest, il s'agit de villas, à I'Est, ce sont des pavillons populaires et dans le Sud de l'habitat bon marché. Dans les années 1950, des immeubles de logements soviétiques y sont disséminés, puis dans les années 1980, les artères principales sont ceinturées d'habitat collectif de moindre qualité. Après 1990, ces quartiers sont les premiers valorisés, en raison de leur proximité du centre.

Les grands ensembles résidentiels des années 1960 et 1970 sont des espaces urbains hétérogènes en raison de la diversité des dimensions, des formes et de la qualité des immeubles. Ces quartiers denses sont inspirés par la "cité radieuse " de Le Corbusier. Ils ont été conçus avec de nombreux espaces verts, de grandes artères et un réseau de transport en commun adapté. Mais faute de ressources, les infrastructures et services qui y étaient prévus n'ont pas toujours été réalisés. Il s'agit aujourd'hui du type de produit le plus accessible dans les itinéraires résidentiels.

Les zones extérieures sont les plus étendues et les plus variées, le nombre de logements collectifs diminuant avec l'éloignement au centre. Elles accueillent les nouveaux arrivants qui y construisent eux-mêmes de petites maisons de style rural, souvent dans des lotissements illégaux, dépourvus de toute viabilité. Ces constructions accroissent l'étalement de la capitale que les autorités ne parviennent pas à endiguer. Sur ces lotissements se sont superposés des petits immeubles de l'Entre-deux-guerres, des groupes de logements soviétiques des années 1950, puis les constructions des années 1960 et 1970 le long des axes menant au centre. Depuis 1990, ces zones ont enregistré le plus grand nombre de constructions, par le remplacement des anciens logements individuels ou la création de nouvelles parcelles aux limites de l'espace résidentiel.

\section{Transfert de propriété, maintien des structures urbaines}

À la fin de la période socialiste, la différenciation spatiale est estompée par la généralisation de I'habitat collectif et les faibles écarts des salaires: la majorité des habitants partagent des conditions de vie semblables et la société roumaine est brassée. Depuis les lois L 61/1990 et L 85/1992 sur la vente des logements propriété de l'Etat aux locataires, les Bucarestois sont devenus dans leur grande majorité propriétaires de leur logement ( $82 \%$ au recensement de 2002). L'apparition d'un marché immobilier transforme et diversifie les structures " homogénéisées » de la 
période précédente. Le centre ville, rendu indésirable par le manque d'investissements et la menace des destructions, tarde pourtant à faire peau neuve.

La percée du gigantesque axe Est-Ouest n'a pas affaibli la structuration héritée Nord-Sud de la capitale, qui lie la capitale à son hinterland. Elle guide aujourd'hui l'évolution des prix du foncier (carte 2), et tire la ville vers le Nord. Les lacs et forêts au Nord, cédés par la famille royale à la ville en 1931 , sont devenus de puissantes aménités pour la capitale. Les palais du tournant du XXème ${ }^{\text {siècle et }}$ les villas cossues de l'Entre-deux-guerres y avaient été réservés à la nomenclature communiste, ils attirent à présent l'élite politique et économique. A l'opposé, au Sud, les quartiers de lotissements populaires, même lorsqu'ils ont été remplacés par des grands ensembles semblables à ceux de l'Est ou de l'Ouest, comme le quartier Rahova, continuent d'accueillir les populations marginalisées.

La prégnance des ces héritages est renforcée par le processus de rétrocession des immeubles nationalisés par les communistes. En Roumanie, ce processus n'est toujours pas achevé ${ }^{4}$. La loi fondamentale de 1991 sur les rétrocessions est contournée par la loi L 112/1995 qui permet la vente aux locataires à des prix très avantageux, même si les logements sont réclamés par leurs anciens propriétaires. Elle est ensuite modifiée sous pression de l'UE par la loi L 10/2001 sur la restitution des biens et immeubles confisqués, qui donne encore aujourd'hui lieu à de nombreux procès et litiges, empêchant une consolidation de la propriété. Dans le centre ville, les imbroglios se sont multipliés. En effet, le front de démolition lié aux opérations d'urbanisme des années 1980 avait été occupé par des populations marginales au fur et à mesure que les anciens occupants étaient chassés par les autorités. Au début des années 1990, le centre ville, très dégradé, est habité par d'importantes communautés Roms. Elles sont à leur tour chassées par les restitutions, tandis que les nouveaux (re)venus, lorsqu'ils ont des ressources suffisantes, stimulent la réhabilitation des immeubles et la spéculation foncière. Toutefois, ces rétrocessions, du fait des litiges, du manque de ressources ou de la spéculation, favorisent les transformations en plombages, le maintien d'immeubles en mauvais état et parfois leur occupation abusive.

\section{De nouvelles dynamiques résidentielles limitées face aux mutations des activités}

La libéralisation du sol urbain profile de nouvelles dynamiques résidentielles. Avant 1990, le sol était propriété de l'Etat, qui avait le monopole de l'aménagement du territoire et de l'urbanisme. L'abrogation rapide des lois et des règlements liés au contrôle de la propriété, de la résidence et de la construction a favorisé les initiatives privées ${ }^{5}$. Cependant, la crise économique conduit à l'arrêt rapide des constructions de logements collectifs. Les constructions nouvelles restent limitées $(2,5 \%$ du parc au recensement de 2002), mais elles vont s'accélérant ${ }^{6}$. De même, les mobilités intraurbaines n'ont concerné entre 1992 et 2002 que 17\% des habitants, soit à peine plus que ceux qui

\footnotetext{
${ }^{4}$ L'Administration du Fonds Immobilier de l'Etat administrait en 2003 plus de 23500 logements à Bucarest, dont 13500 maisons nationalisées et, en 2006, plus de 11000 logements, dont 4500 maisons nationalisées. En 1989, l'Etat était propriétaire de 455000 logement à Bucarest, ce qui représentait alors $69,6 \%$ du fonds existant.

${ }^{5}$ CINÀ G., 2005, Bucarest, dal villaggio alla metropoli. Identità urbana e nuove tendenze, Edizioni Unicopli, Milan, pp. 149150

${ }^{6}$ En 2000, environ 26000 autorisations de construire ont été délivrées à Bucarest pour des bâtiments d'habitation, plus de 27600 en 2003 et près de 46000 en 2007, dont près de 97\% pour des logements individuels (selon l'Institut National de Statistique). Les lois L 114/1996 et L 62/2006 accordent des aides et subventions à la construction, et le développement du crédit hypothécaire après 2002 fluidifie l'accès au marché immobilier. Toutefois, le maximum observé date 1990, année où tout était possible et les contrôles inopérants, avec 48599 permis de construire accordés pour des logements.
} 
ont quitté la capitale pendant la même période. Dans un contexte de diminution et de vieillissement de la population ${ }^{7}$, la stabilité résidentielle apparente ne traduit pas la satisfaction des habitants. Depuis 1990, quitter les immeubles collectifs, les "blocs", abandonner le mode de vie (blocuire) qui y est attaché, est le meilleur symbole de la réussite professionnelle et économique. La dégradation de l'habitat collectif, les nouvelles constructions et la libération de nombreux logements du fait de la diminution de la population ont produit une fuite résidentielle des moins pauvres. Ceux qui ne peuvent pas quitter l'habitat collectif cherchent à se rapprocher du centre ou à rénover leurs appartements. Les " blocs " changent sous l'impulsion des associations de propriétaires ; l'apparition de compteurs individuels d'eau, d'électricité, les tentatives de privatisation des parties communes montrent que l'individuel y prend également pied sur le collectif.

La stabilité résidentielle de la majeure partie des habitants est le reflet surtout d'une situation subie, du fait de l'explosion des prix immobiliers, mais aussi du manque de solutions plus intéressantes. Cependant, le rythme des constructions est très faible, en 2004 seule 1725 logements on été livrés, avec une superficie moyenne de $152 \mathrm{~m}^{2}$, ce qui explique pourquoi les Bucarestois ont du mal à trouver un logements correspondant à leurs projets. Seulement $30 \%$ des Bucarestois interrogés ${ }^{8}$ prévoient de changer de logement, ils habitent pour plus d'un tiers dans des deux pièces. Ces projets sont envisagés à moyen terme (37\% dans les 5-10 années suivantes) et visent un logement plus grand (43\%) ou un logement individuel (38\%). Les occupants de maisons individuelles, quel qu'en soit le type, ne formulent pas de projet de mobilité. Ces aspirations révèlent une nouvelle hiérarchie d'attractivité ou de répulsion des différents quartiers de Bucarest. Les quartiers en cours de rénovation du centre ville, les villas des zones intermédiaires et les complexes résidentiels extérieurs sont les produits les plus prisés. La proportion réduite de nouveaux arrivants confirme que les grands ensembles extérieurs sont en situation de fin de trajectoire résidentielle. Cependant, la répulsion des immeubles collectifs n'est pas absolue, certains immeubles bien placés peuvent garder un certain attrait, d'autant qu'ils sont les plus abordables. À Bucarest, après les renversements des hiérarchies sociales liés à la "transition ", la tendance à exhiber sa réussite est très forte. La valeur associée à un logement dépend de sa localisation et du statut social du voisinage, ce qui contribue à différencier fortement les quartiers. Pourtant, il existe des logements confortables dans des quartiers dégradés et des logements de faible qualité dans des quartiers partiellement réhabilités, ce qui empêche de faire des hiérarchies linéaires.

Ces évolutions lentes contrastent avec la rapidité des remodelages engendrés par le double mouvement de désindustrialisation et de tertiarisation. Aujourd'hui, Bucarest compte 10 centres commerciaux et plus de 2 millions de mètres carrés de bureaux. La construction de bureaux connaît une croissance exponentielle ${ }^{9}$ et conserve un fort potentiel puisque le taux d'inoccupation reste inférieur à $1 \%$ des surfaces. La désindustrialisation s'accélère ${ }^{10}$ et les plates-formes industrielles héritées sont laissées en friche. Depuis 1996, plus de 150 ha de friches ont été requalifiés, soit plus de 10\% de l'emprise industrielle. La mairie a bénéficié des fonds Phare de l'UE entre 2003 et 2005

\footnotetext{
${ }^{7}$ Entre 1992 et 2005, Bucarest perd près de 200000 habitants; sur la même période, la part des plus de 70 ans passe de $6,75 \%$ à plus de $10 \%$ selon l'INS.

${ }^{8}$ SUDITU B., 2006, Logements, habitants et mobilités résidentielles à Bucarest, thèse des Universités d'Angers et de Bucarest.

9 Entre 2004 et 2006, 150000 mètres carrés de bureaux sont livrés annuellement à Bucarest, $410000 \mathrm{~m}^{2}$ en 2007 et $350000 \mathrm{~m}^{2}$ sont prévus pour 2008 (INS, 2007).

${ }^{10}$ Au milieu des années 1990, les plates-formes industrielles voient leur activité réduite, jusqu'à moins d'un tiers. Ces 10 dernières années, le total des emplois industriels hors construction est passé à Bucarest de 274000 à 160000 (INS, 2007).
} 
pour accompagner ces mutations foncières (dépollution, réseau d'assainissement) dans le Nord de Bucarest. La mutation des espaces industriels commence autour des stations de métro, qui avaient été réalisées pour acheminer les ouvriers depuis les grands ensembles. Dans l'Est de la capitale, les 36 ha des usines textiles et métallurgiques autour de la station de métro Republica ont laissé place à la grande distribution (Cora, Mobexpert, Bricostore). Dans l'Ouest, la grande distribution (Carrefour, Mobexpert, IDM) occupe 15 ha à proximité de la gare Basarab, à la place terrains vagues et d'une partie de la régie des tabacs. Plus au Sud, la plate-forme Semanatoarea est encadrée par deux stations de métro, d'un côté, ses bâtiments sont convertis en espaces commerciaux, de l'autre, les friches laissent place à des bureaux, comme Sema Parc $\left(43000 \mathrm{~m}^{2}\right)$, dont les deux immeubles devraient être livrés à la mi 2008. Les nouveaux projets se concentrent à présent dans le Nord, en direction des aéroports, mitant les terrains agricoles et les forêts.

\section{Un étalement urbain vers le Nord de la capitale}

Les constructions se concentrent dans le Nord, surtout à proximité des lacs et sur les rives de la Colentina, aux dépens des espaces verts, des terrains agricoles et des forêts. Cette reprise de l'étalement se conjugue avec une perte d'habitants, alors que les quartiers Nord étaient déjà les moins denses. La recherche d'une meilleure qualité de vie, se révèlent souvent un trompe-l'œil : le manque de plan d'ensemble se traduit par une forte densité de logements sans trame viaire ni réseaux, dépendant de puits pour leur alimentation alors que les eaux souterraines sont très polluées et déversant leurs eaux usées dans les lacs. Même si l'autoconstruction à l'écart de toute viabilité cède la place aux investisseurs publics et privés, cela se fait sans amélioration notoire de la qualité de vie. Ainsi, l'Agence Nationale pour le Logement a lancé en 2006 la construction de plus de 1000 maisons dans le cadre d'un programme d'accession à la propriété, sur un terrain de 99 ha qui longe la piste de l'aéroport de Baneasa. Les maisons, qui seront livrées à la fin 2008 , verront passer les avions à moins de 100 mètres. Les forêts sont par ailleurs défrichées à proximité de l'aéroport entre 2006 et 2007 pour créer près de 100 ha de nouveaux espaces commerciaux et de bureaux.

Le Nord de la capitale est synonyme de prestige, comme le traduisent les « résidences fermées ${ }^{11}$ ". Ce type de produit apparaît à Bucarest en 1994 avec le "Village français ». Ce complexe de 34 villas et 35 appartements, construit par Bouygues à proximité des lacs, est clôturé et comprend des services sportifs, de santé, de gardiennage et de loisirs. Il vise une population d'expatriés cherchant le calme et la sécurité, mais il n'est pas une " communauté fermée " puisqu'il propose ses produits uniquement à la location. Ce modèle se diffuse ${ }^{12}$, en 2007 les complexes résidentiels fermés représentent près de 3000 villas à la location ou à la vente, toutes concentrées dans le Nord de Bucarest et sur la commune voisine de Voluntari, entre les lacs Colentina, l'aéroport et la forêt de Baneasa.

\footnotetext{
${ }^{11}$ RUFAT S., 2004, « Les 'résidences fermées' à Bucarest : de l'entre-soi à la fragmentation ? ", Arches, n 6, pp. 114-121

${ }^{12}$ En 1995, la société Impact SA suit cet exemple en lançant un projet de complexe résidentiel, "Alpha », dont les 44 villas de $350 \mathrm{~m}^{2}$ avec piscines et court de tennis au sein d'une même clôture finissent d'être construites en 2001. Il est suivit de " Bêta », " Gamma », « Delta », « Epsilon » et de projets d'autres promoteurs.
} 


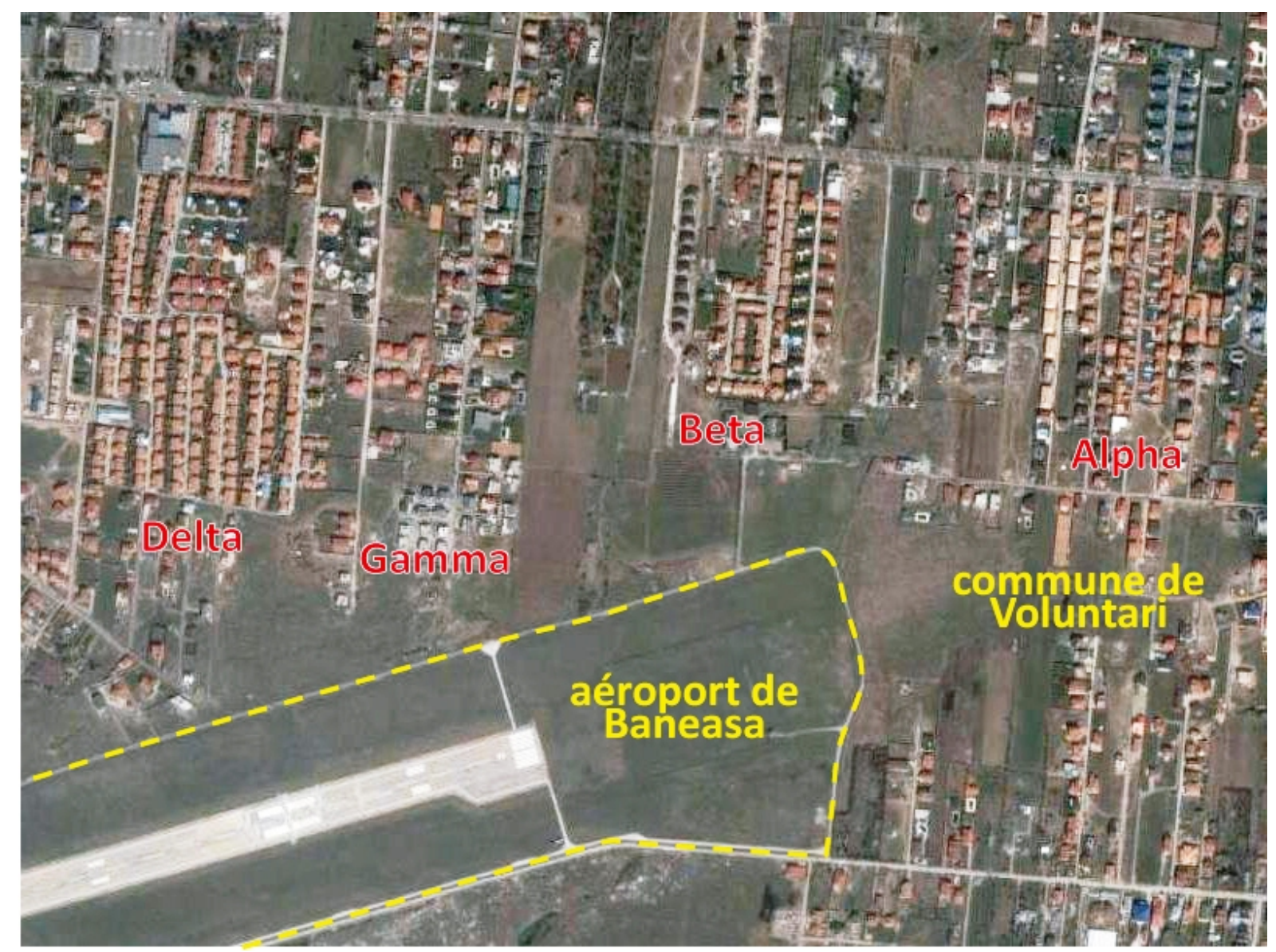

Image : Les résidences fermées au bord de la piste de l'aéroport de Baneasa (2007).

La libéralisation du marché et la spéculation, alimentée par l'argent des Roumains partis travailler à l'étranger, font exploser le marché immobilier, dont les prix doublent entre 2003 et 2006, dans la perspective d'adhésion à l'UE (carte 2). L'accès à la propriété des nouveaux ménages devient presque impossible, au moment où l'Etat restreint la construction de logements sociaux. Les litiges liées aux restitutions et la spéculation foncière favorisent l'étalement urbain de la capitale : loin du centre, les terrains sont abordables et leur statut moins incertain. Cet étalement est un trait essentiel de Bucarest qui reste une ville "basse ", à la croissance plus horizontale que verticale. Les autorités qui avaient cherché à l'endiguer dès le début du XX⿳亠丷⿵冂丶 ${ }^{2}$ siècle, sans y parvenir, s'y trouvent à nouveau confrontées, avec les coûts édilitaires que cela implique.

\section{Les tâtonnements de l'urbanisme}

Face à ces nouvelles dynamiques économiques et résidentielles, face aux mutations de l'espace urbain, les autorités locales sont restées longtemps impuissantes. Les centres commerciaux et d'affaires, les parkings et tours de bureaux surgissent en partie au hasard des opportunités foncières, en dehors de la requalification des friches industrielles. Mais la logique de valorisation foncière pousse également les acteurs à construire sur les terrains en litige ou dans les espaces verts. Le manque de nouveaux documents d'urbanisme assurant une cohérence à l'échelle de la capitale conduit la mairie à autoriser les opérations au cas par cas, sans vision d'ensemble ${ }^{13}$. Les années 1990

\footnotetext{
${ }^{13}$ CLAVEL M., 1998, "Construire l'espace urbain à Bucarest », Géographie et Cultures, n²7, pp. 115-130
} 
voient donc réapparaître les problèmes récurrents de la capitale : les constructions aux marges de la légalité et l'étalement incontrôlée des tissus. La politique du fait accompli est d'autant plus efficace que les destructions et les déclarations d'utilité publique rappellent les pratiques de l'ancien régime. Ces constructions entraînent de nouveaux conflits quand les immeubles de très grande hauteur envahissent les espaces verts ou masquent les héritages patrimoniaux et les édifices religieux (Cathédrale catholique, Eglise arménienne), ce qui rappelle également le mode opératoire de l'ancien régime.

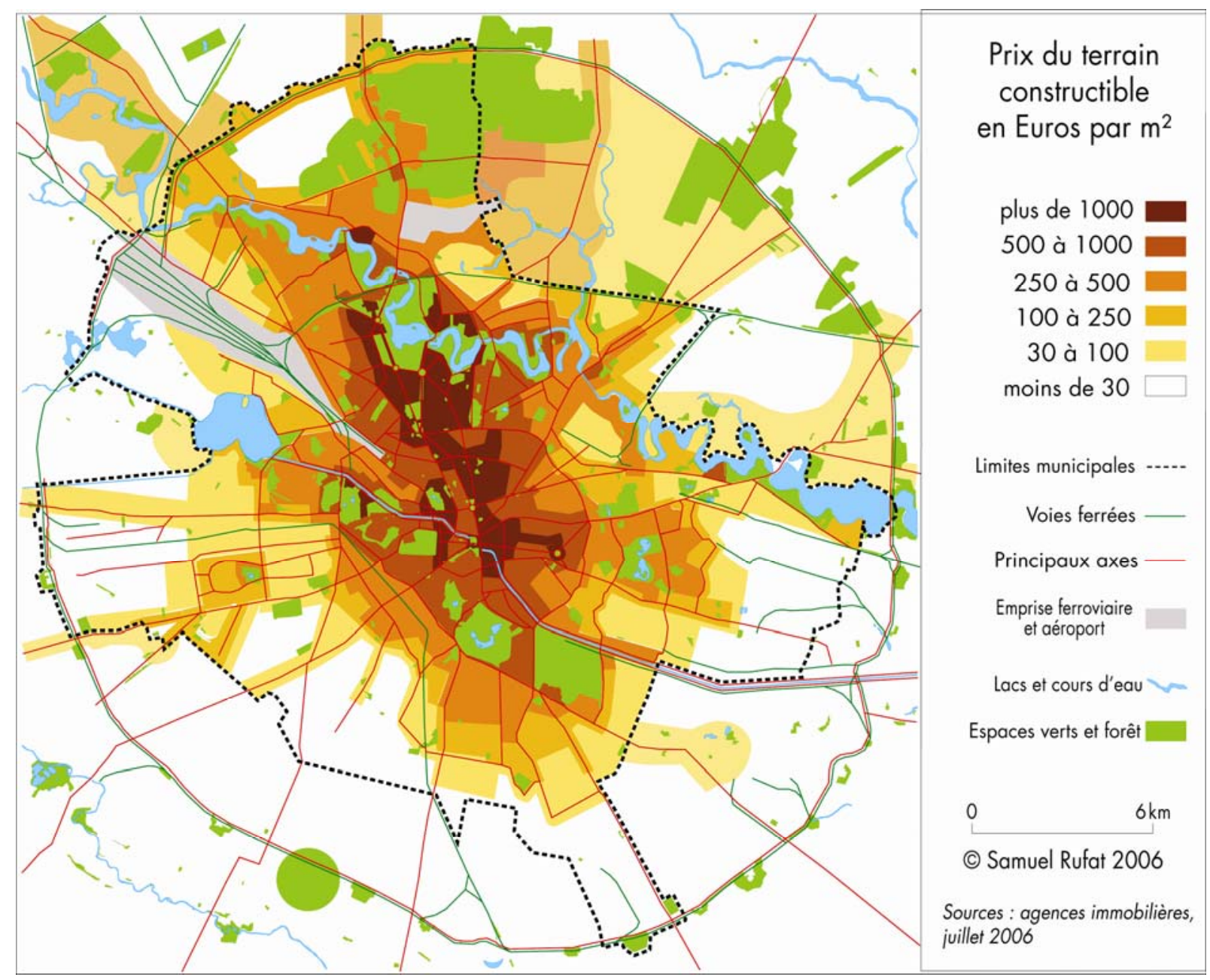

Carte 2 : Prix du terrain constructible en 2006

En revanche, les trois principaux projets de revitalisation de la capitale, concentrés dans le centre ville, ne se sont toujours pas matérialisés. II s'agit tout d'abord de du projet " Bucarest 2000 ", qui a donné lieu à un concours international en 1995, devait faire entrer la ville dans la modernité européenne en digérant le lourd héritage des travaux inachevés de Ceausescu. Il prévoyait d'intégrer le Centre Civique aux tissus existants par la création d'un centre d'affaire et de tours. Incroyable pour un projet de cette ampleur, il est resté lettre morte, du fait des litiges de propriété et des opportunités biens plus intéressantes sur les friches contestées du centre ou vers le Nord. Ensuite, la réhabilitation du centre historique, dont une partie des rues pavées sont devenues interdites à la circulation, qui reste en grande partie portée par l'initiative privée. Une "zone historique de référence " a été délimitée en 2001 (OG 77/2001) pour stimuler les travaux en partenariat public/privé. Mais le périmètre restreint retenu ( 50 ha) et les litiges de propriété ne favorisent pas les 
opérations de grande ampleur ${ }^{14}$. Enfin, le projet "Esplanada » prévoit la construction d'un autre centre d'affaire de 10 ha à l'autre bout du nouvel axe Est-Ouest, à la place des chantiers suspendus. II $s^{\prime}$ agit du troisième projet consécutif ${ }^{15}$ sur cet espace qui semble encore abandonné, les travaux devraient commencer courant 2008.

Un Plan d'Urbanisme Général existe bien, il a été réalisé par l'Université d'Architecture en 1998, il a été adopté en 2001, mais il ne contient aucun de ces projets. Etabli au $10000^{\mathrm{e}}$, ce plan est complété par des règlements d'utilisation du sol au $5000^{\circ}$, qui reposent sur une mise à jour des documents antérieurs à la période socialiste. Les documents d'urbanisme précisent le type d'occupation du sol, les zones fonctionnelles, l'altimétrie et l'alignement des constructions, répondant aux carences du début des années $1990^{16}$. Mais ils ne proposent pas de stratégie de développement à moyen terme, de lignes de forces permettant de canaliser l'extension des tissus ou de réserves foncières. Le plan d'urbanisme se fait parfois héritier des projets de la ville socialiste ${ }^{17}$.

Les plans des urbanistes ne se font pas seulement la caisse de résonance des différents héritages de la capitale, ils proposent d'appliquer par mimétisme les grands thèmes de l'urbanisme occidental de la fin du XXème siècle à Bucarest ${ }^{18}$. Les analyses montrant la nécessité de la métropolisation ou des requalifications foncières commencent toutes par un argument irréfutable : "c'est ce qui se fait dans les autres capitales européennes ». En fait, trois projets différents de création d'une Zone Métropolitaine de Bucarest sont mis en route : I'un par l'Institut de Géographie, un autre par le Centre de Planification Urbaine et Métropolitaine de Bucarest, et le dernier par le maire du premier arrondissement de la capitale ${ }^{19}$. Leurs découpages varient selon les critères retenus de 30 à 88 communes, voire l'intégralité du département Ilfov qui entoure la capitale. Cependant, aucun d'entre eux ne donne de contenu ni de budget à cette nouvelle maille. Ils montrent que le mimétisme conduit à la création de formes sans projet : faute de volonté politique ils n'aboutiront pas. Les maires des communes jouxtant Bucarest au Nord et à l'Est (Otopeni, Voluntari, Popesti Leordeni) ont déjà refusé de participer à une zone métropolitaine, quel que soit le périmètre retenu.

Le Plan d'Urbanisme Général souffre d'une volonté de flexibilité, en plus du manque d'arbitrage politique entre héritages et mimétisme. Dans les règlements, une catégorie très vaste, " fonction mixte jusqu'à 14 étages " a été largement utilisée. Alors que la loi, de portée nationale, prévoit que les plans de détail soient réalisés en concordance avec le plan général (L 215/2001 art. 95), à Bucarest, les règlements municipaux donnent souvent la primauté aux plans de détail. Dans la

\footnotetext{
${ }^{14}$ HAPENCIUC BLUM I., 2007, Régénération urbaine et renouvellement des pratiques d'urbanisation en Roumanie : Le cas du centre historique de Bucarest, thèse de l'INSA de Lyon, sous la direction de M. Zimmermann

${ }^{15}$ Lorsque le Parlement s'est installé dans le Centre Civique, rebaptisé «Palais du Peuple ", d'abord la Chambre de députés en 1996 puis le Sénat en 2004, le gouvernement avait réclamé le bâtiment inachevé de la bibliothèque centrale qui fait face au "Palais du Parlement ". Suite aux conflits juridiques, ce bâtiment inachevé est resté dans l'état où l'a surpris la Révolution de décembre 1989 , et ses grues, rouillées, ont commencé à tomber.

${ }^{16}$ Dutu M., 2004, Dreptul urbanismului. Teorie si practica judiciara, Ed. Economica, Bucarest, pp. 101-108

${ }^{17}$ Le Plan d'Urbanisme Général reprend par exemple la construction du centre socioculturel sur les marges du parc Titan, qui n'avait pu être réalisé en même temps que les grands ensembles socialistes dans les années 1970, faute de ressources.

${ }^{18}$ Ce mimétisme est la plupart du temps assumé, même s'il repose parfois sur une analyse simpliste. Sur le site du maire de Bucarest, on pouvait lire en 2007 : "En Europe, les déchets sont traités comme une mine d'or. Triés et recyclés, les déchets se transforment en source de matières premières, d'énergie bon marché et d'emploi biens rémunérés. La solution est que la mairie stimule le tri des déchets par catégories " www.videanu.ro

${ }^{19}$ Rey V., Groza O., Ianos I., PATroescu M. (dir), 2007, Atlas de la Roumanie, Montpellier, CNRS GDRE S4 - La Documentation Française, p. 171
} 
pratique, la mise en cohérence des différentes opérations reste donc assez difficile. D'autant plus que les infractions aux normes d'urbanisme sont sanctionnées par des amendes dérisoires ${ }^{20}$ et que suite aux traumatismes de l'urbanisme de Ceauşescu, il est difficile de détruire même les édifices illégaux, insalubres, ou endommagés.

Ces tâtonnements et dysfonctionnements deviennent un handicap important, en raison de la spéculation mais aussi de l'intégration européenne. Alors que la loi ne prévoit pas la constitution de réserves foncières, les restitutions ont largement entamé celles de la municipalité et chaque nouveau projet est renchéri par la spéculation foncière. De plus, les Fonds Structurels et Régionaux Européens auxquels Bucarest est éligible, ne peuvent être obtenu sans un plan de développement stratégique à moyen terme et un budget pluriannuel, qui n'ont pas été réalisés pour la capitale. Enfin, la mairie n'a pas été en mesure d'absorber les crédits obtenus ces dernières années, comme les prêts non remboursables proposés en 2005 par le Gouvernement des Pays-Bas pour la réhabilitation du centre historique. Certains projets parviennent cependant à voir le jour, comme le pont Basarab, le chantier public le plus important à Bucarest depuis 1989. Ce pont de près de $2 \mathrm{~km}$ doit enjamber la Dâmbovița et l'importante emprise ferroviaire de la gare Basarab. II est le dernier tronçon de l'anneau de circulation intérieure, qui doit délester le centre ville saturé par la circulation ${ }^{21}$. Le projet obtient un financement de 68 millions d'Euros de la Banque Européenne d'Investissement en 2000, mais le dossier est clos en 2002, suite aux divergences entre le maire et le conseil municipal de Bucarest. Le projet est relancé début 2006, mais bute sur la nécessaire expropriation de $50000 \mathrm{~m}^{2}$ de terrain, dont le prix a plus que doublé (jusqu'à $1500 \mathrm{Euros} / \mathrm{m}^{2}$ ), ce qui porte le coût total à 225 millions d'Euros. La mairie fait un appel à l'épargne pour le financer, elle n'a pour l'instant pas obtenu d'autre financement européen. Les travaux ont commencé en octobre 2006, ils devraient finir début 2009.

\section{Une gestion dépendante et sur le court terme}

Les modes de gouvernance morcelée à l'échelle métropolitaine ne favorisent pas une gestion cohérence ou l'émergence de politiques ambitieuses. Le découpage radial de la capitale en six arrondissements (secteurs) date de 1981, Bucarest est devenu un département après 1989. Ce maillage correspond à une gestion encore assez centralisée qui ne facilite pas la coopération avec les communes voisines. La répartition des attributions entre le maire général, le conseil municipal général, les six maires d'arrondissement, les six conseils municipaux et le préfet nommé par le gouvernement est prévue par la loi sur l'administration publique locale (L 215/2001). Cette loi affirme le principe de décentralisation mais donne lieu à des interprétations divergentes. Le mode de scrutin, qui prévoit un quadruple suffrage direct, séparant l'élection des maires et des conseils aux deux échelons, favorise les cohabitations et les conflits politiques internes, entre les maires et les

\footnotetext{
${ }^{20}$ L'immeuble Tower Center International, à 200 mètres de la Place de la Victoire et du Gouvernement, à côté de la tour construite en 2005 pour le groupe BRD-Société Générale, a obtenu en 2006 un permis de construire. Il prévoyait 21 étages de bureaux et 3 niveaux de souterrain, ce qui devait en faire l'un des plus haut de la capitale. En février 2007, la mairie constate qu'un étage et un niveau souterrain supplémentaires ont été réalisés, elle sanctionne l'infraction d'une amende de 10000 Lei nouveaux (moins de $3000 €$ ). Mais les promoteurs, Arvig 35 et Alpha Bank, ne sont pas obligés de renoncer aux étages supplémentaires. Les étages sont entérinés par un "projet d'entrée en légalité ", qui modifie les documents d'urbanisme. Le prix du mètre carré de bureau à la location dans ce quartier prisé (entre 35 et $40 €$ par mois), rend le montant de cette amende dérisoire pour un bâtiment d'environ $22000 \mathrm{~m}^{2}$ de bureaux, qui sera livré début 2008.

${ }^{21}$ Le nombre de véhicules particuliers immatriculés à Bucarest est passé de 370000 en 1995 à plus de 800000 en 2006, alors que la population a décru de 2,05 à 1,9 millions d'habitants sur cette période (INS, 2007).
} 
conseils d'une part, entre les équipes des différents secteurs et la mairie générale d'autre part. De plus, le budget municipal dépend à plus de $60 \%$ du versement par l'Etat d'une partie des impôts sur le revenu (tableau). Les dépenses de fonctionnement représentent $85 \%$ du budget municipal. Pour pouvoir investir, alors qu'elle elle perd au $1^{\mathrm{er}}$ janvier 2008 les taxes sur les transactions immobilières, qui sont reversées au budget de l'Etat, et une partie de la TVA qui lui était reversée, la mairie générale a prévu de doubler en 2008 son recours à l'emprunt.

\begin{tabular}{|c|c|c|c|c|c|c|c|}
\hline & $\begin{array}{c}2006 \\
\text { (en millions } \\
\text { de Lei } \\
\text { nouveaux) }\end{array}$ & $\%$ & $\begin{array}{c}2007 \\
\text { (en millions } \\
\text { de Lei } \\
\text { nouveaux) }\end{array}$ & $\%$ & $\begin{array}{c}2008 \\
\text { (estimations) }\end{array}$ & $\%$ & $\begin{array}{c}2008 \\
\text { (estimations } \\
\text { en millions } \\
\text { d'Euros } \\
\text { janvier 2008) }\end{array}$ \\
\hline $\begin{array}{c}\text { Impôts } \\
\text { locaux }\end{array}$ & 164,3 & $6,27 \%$ & 327,3 & $8,50 \%$ & 243,5 & $5,01 \%$ & 73,8 \\
\hline $\begin{array}{c}\text { Cote part } \\
\text { reversée des } \\
\text { impôts sur } \\
\text { le revenu }\end{array}$ & 1623,5 & $61,94 \%$ & 2360,8 & $61,32 \%$ & 2930 & $60,25 \%$ & 887,9 \\
\hline $\begin{array}{c}\text { Cote part } \\
\text { reversée de } \\
\text { la TVA }\end{array}$ & 130,7 & $4,99 \%$ & 380,2 & $9,88 \%$ & 81,4 & $1,67 \%$ & 24,7 \\
\hline $\begin{array}{c}\text { Revenus } \\
\text { propres }\end{array}$ & 33,0 & $1,26 \%$ & 33,1 & $0,86 \%$ & 41,5 & $0,85 \%$ & 12,6 \\
\hline $\begin{array}{c}\text { Fonds de } \\
\text { roulement }\end{array}$ & 90,7 & $3,46 \%$ & 94,0 & $2,44 \%$ & 137,2 & $2,82 \%$ & 41,6 \\
\hline Emprunt & 578,6 & $22,08 \%$ & 654,2 & $16,99 \%$ & 1429,1 & $29,39 \%$ & 433,0 \\
\hline TOTAL & $\mathbf{2 6 2 0 , 9 5}$ & $100,00 \%$ & $\mathbf{3 8 4 9 , 7}$ & $100,00 \%$ & $\mathbf{4 8 6 2 , 7}$ & $100,00 \%$ & $\mathbf{1 4 7 3 , 6}$ \\
\hline
\end{tabular}

Budget de la mairie générale de Bucarest, recettes (source : PMB)

Les maires d'arrondissement cherchent alors à mettre en place des politiques "visibles" avec le budget dont ils disposent, c'est-à-dire à court terme. Ainsi, plus que la modification des structures héritées ou le développement de politiques à moyen terme, ce sont les places de parkings et les espaces verts qui préoccupent les maires. La politique de "discipline dans la construction » ( L 50/1991 et L 117/2007) cherche pourtant à résorber les constructions illégales, notamment dans les espaces verts et espaces publics. Depuis 1990, en réaction à la période de collectivisation, la privatisation de l'espace public a permis le développement des services de proximité qui faisaient défaut aux quartiers résidentiels. Les espaces verts entre les immeubles ont eux aussi souvent été transformés selon les besoins en vergers, parkings ou dépôts, ce qui peut être lu comme une nouvelle manifestation du Bucarest « villageois ${ }^{22} »$.

A partir de la campagne des élections municipales de 2004, les pouvoirs urbains ont mené une reconquête de ces différents espaces publics ou semi publics, avec des interventions très médiatisées dans le quartier Drumul Taberei (grands ensembles de l'Ouest). Tout d'abord, les kiosques et les boutiques improvisés sur les trottoirs, l'un des emblèmes des paysages de la transition, ont été démolis. Puis les mairies d'arrondissements se sont attaquées aux constructions plus résistantes, les parkings en béton préfabriqué et les boutiques en structures métalliques. Les

\footnotetext{
${ }^{22}$ Vossen J., 2004, Bukarest. Die Entwicklung des Stadtraumes, Dietrich Reimer Verlag, Berlin, p. 288 et 292
} 
constructions illégales ont été remplacées par du mobilier urbain " moderne " (abribus, bancs, kiosques à journaux). Depuis 2004, le processus de reconquête de l'espace public s'est généralisé.

\section{Conclusion}

Bucarest continue de souffrir de son rôle de capitale, les autorités ont toujours cherché à transformer en vitrine de la modernité l'ancien "conglomérat de villages" parsemé de caravansérails et de monastères. Aujourd'hui, le traumatisme encore récent de l'urbanisme de Ceausescu ne permet pas d'envisager une nouvelle transformation de grande ampleur. Les remodelages restent avant tout locaux, au hasard des marges de manœuvres laissés par les contentieux juridiques et la spéculation foncière. Les héritages incomplètement maîtrisés accentuent la lenteur des mutations, rappelant l'analyse de Vintila Mihailescu qui déclarait au début du XXème siècle que c'est " une ville qu'il n'est pas aussi facile de transformer qu'on pourrait le croire au premier abord ${ }^{23}$ ». En l'absence de projet à moyen terme et sans voie propre de développement, Bucarest semble condamnée à un mimétisme servile des modèles occidentaux. Le maire actuel s'était pourtant fixé pour objectif de renforcer l'identité de la capitale. II propose d'accompagner la réhabilitation du centre ville et de reprendre deux projets du début du XXème siècle : une ceinture verte, sur le modèle londonien, et la construction d'une cathédrale, dont la tradition orthodoxe est dépourvue.

Les traits caractéristiques de Bucarest (ville basse, polycentrique, végétalisée), auraient cependant pu être mis en valeur en profitant des nombreuses friches du centre ville et de l'inachèvement de l'urbanisme de Ceausescu. La concentration des tours de bureaux en un nouveau quartier d'affaires autour du Palais du Parlement aurait permis un autre traitement de ces friches. Elles ne doivent pas être considérées comme des espaces vides à remplir rapidement. Elles auraient pu servir à briser le bâti continu le long des grands axes qui masque les tissus anciens, à articuler les différentes strates urbaines tout en canalisant les aspects "villageois". A une autre échelle, la tendance lourde à l'étalement vers le Nord serait contrebalancée en améliorant l'équipement des quartiers Sud. Le Sud de la métropole gagnerait à être considéré comme une réserve foncière et accueillir d'importantes infrastructures, ce qui orienterait enfin Bucarest vers le Danube.

Le manque de choix politiques et de projets stratégiques se révèle être un handicap à l'heure de l'intégration européenne. La capitale ne semble pas prête, plus d'un an après l'adhésion, à répondre aux critères de qualité européens ou à en capter efficacement les financements. Ces nouvelles exigences liées aux standards de l'UE sont à la fois une chance et un défi pour Bucarest de se donner les moyens de ses ambitions.

\section{Références}

CINÀ G., 2005, Bucarest, dal villaggio alla metropoli. Identità urbana e nuove tendenze [Bucarest, du village à la métropole. Identité urbaine et nouvelles tendances], Edizioni Unicopli, Milan, 178 p.

DANTA D., 1993, "Ceaucescu's Bucharest », Geographiacl Review, vol. 83, n², pp. 170-182

DUTU M., 2004, Dreptul urbanismului. Teorie si practica judiciara [Droit de l'urbanisme. Théorie et pratique judiciaire en Roumanie], Ed. Economica, Bucarest, 360 p.

\footnotetext{
${ }^{23}$ Mirailescu V., 2003, op. cit., p. 15
} 
HARHOIU D., 1997, Bucarest, une ville entre Orient et Occident, édition bilingue français et roumain, Simetria-Arcub, Bucarest, 135 p.

INS - Direcția Regională de Statistică a Municipiului Bucureşti, 2007, Bucharest. Statistical Yearbook, édition bilingue anglais et roumain, Bucarest, INS, $152 \mathrm{p}$.

MiHAILESCU V., 2003, Bucureşti. Evoluția geografică a unui oraş [Bucarest. Evolution géographique d'une ville], Paideia, Bucarest, $365 \mathrm{p}$.

RUFAT S., 2004, "Les résidences fermées à Bucarest : de l'entre soi à la fragmentation ? ", Arches, Revue Internationale de Sciences Humaines, Tome 6, pp. 83-93

SUDITU B., 2006, Logements, habitants et mobilités résidentielles à Bucarest, thèse des Universités d'Angers et de Bucarest, $314 \mathrm{p}$.

VOSSEN J., 2004, Bukarest. Die Entwicklung des Stadtraumes [Bucarest. Le développement de l'espace urbain], Dietrich Reimer Verlag, Berlin, 320 p. 